\title{
Spotlight on Trastuzumab Deruxtecan (DS-820I, T-DXd) for HER2 Mutation Positive Non-Small Cell Lung Cancer
}

\author{
Ibrahim Azar (D) \\ Samer Alkassis ${ }^{2}$ \\ Jami Fukui ${ }^{3}$ \\ Fares Alsawah' \\ Kalub Fedak' \\ Mohammed Najeeb Al Hallak' \\ Ammar Sukari (iD) \\ Misako Nagasaka (iD) ${ }^{1,4}$ \\ 'Department of Oncology, Wayne State \\ University School of Medicine, Karmanos \\ Cancer Institute, Detroit, MI, USA; \\ ${ }^{2}$ Department of Internal Medicine, \\ Wayne State University School of \\ Medicine, Detroit, MI, USA; ${ }^{3}$ University \\ of Hawaii Manoa Cancer Center, John \\ A. Burns School of Medicine, Honolulu, \\ HI, USA; ${ }^{4}$ Division of Neurology, \\ Department of Internal Medicine, \\ St. Marianna University School of \\ Medicine, Kawasaki, Kanagawa, Japan
}

\begin{abstract}
Human epidermal growth factor receptor 2 (HER2) is a proto-oncogene that, when mutated or overexpressed, plays an important role in oncogenesis. The landscape of HER2-positive breast cancer has changed dramatically over the past 2 decades with the FDA approval of a growing number of agents (antibodies, tyrosine kinase inhibitors, and antibody-drug conjugates) targeting the HER2 receptor. HER2 inhibition has also been approved for HER2-positive gastric cancer. HER 2 is amplified in $9 \%$ and mutated in $3 \%$ of lung cancer. Historically, HER2-targeted therapy for lung cancer with trastuzumab, pertuzumab, and trastuzumab emtansine has failed to demonstrate a survival benefit. Trastuzumab deruxtecan (T-DXd) is a novel antibody-drug conjugate with a tetrapeptide linker, which delivers a topoisomerase I inhibitor with a drug-to-antibody ratio of 7 8. The potency of the active payload, as well as its significant bystander effect, resulted in significant anti-tumor activity. The DESTINY-Lung01 trial evaluated T-DXd in HER2-positive non-squamous non-small cell lung cancer (NSCLC) and reported a progression-free survival of 14 months in HER2-mutated NSCLC, earning its breakthrough designation by the FDA. In this review, we will discuss the structural characteristics, pharmacodynamics, and pharmacokinetics of T-DXd. We will also shed light on the preclinical and ongoing clinical trials of T-DXd along with future directions in the management of $H E R 2$ positive lung cancer.
\end{abstract}

Keywords: T-DXd, DS8201, antibody drug conjugate, HER2

\section{Introduction}

Human epidermal growth factor receptor 2 (HER2) is a transmembrane glycoprotein receptor with intracellular tyrosine kinase activity, ${ }^{1}$ belonging to the epidermal growth factor receptor $(E G F R)$ family. It is encoded by $E R B B 2$ gene on chromosome 17. Activation of this receptor tyrosine kinase family triggers a cascade of subcellular signal transduction pathways controlling epithelial cell growth, differentiation, motility, and likely angiogenesis in several cell lineages ${ }^{2-5}$ (Figure 1). Overexpression of HER2 activates the phosphoinositide 3-kinase/protein kinase B ( $P I 3 K / A k t)$ pathway, favoring cell proliferation by inhibiting apoptosis. Besides its potentiation as a proliferative effect, HER2 contributes to metastasis by promoting secretion of the matrix metalloproteases and up-regulating specific angiogenic factors, including vascular endothelial growth factor $(V E G F) .^{6-8}$

\section{HER2 in Malignancy}

HER2 can be activated through amplification or mutation. ${ }^{9}$ Overexpression of HER 2 plays a central role in pathogenesis of about $30 \%$ of breast cancers ${ }^{10}$ and 
has been the poster child for success in the development of targeted drug therapy. In non-small cell lung cancer (NSCLC), HER2 overexpression has been implicated in approximately $20 \%$ of adenocarcinoma subtypes. Additionally, HER2 dysregulation has been identified as a mechanism of resistance in EGFR tyrosine kinase inhibitor (TKI) therapy targeted with osimertinib. ${ }^{11} H E R 2$ amplification, as an EGFR-independent mechanism, has been identified in $5 \%$ and $2 \%$ of patients with acquired resistance to second- and first-line osimertinib, respectively. ${ }^{12,13}$ The familial relationship between $E G F R$ and HER2 and their potential to heterodimerize (Figure 1) provides an elegant rationale for the development of resistance.

\section{HER2 Testing}

Tissue testing for overexpression includes immunohistochemistry (IHC) and fluorescent in situ hybridization (FISH) for amplification. HER2 mutation, usually comprising an in-frame insertion in exon $20,{ }^{14}$ is assessed by next-generation sequencing. ${ }^{15}$ Patterns for HER2 overexpression by IHC are scored as IHC 0/1+ (negative/low), IHC $2+$ (equivocal) or IHC $3+$ (positive). For FISH assessment, positivity for HER2 amplification is defined by a HER 2:CEP17 (centromere probe 17 ) ratio of $\geq 2 .{ }^{16}$ In adenocarcinoma of the lung, HER 2 protein overexpression, defined as moderate or strong $(2+/ 3+)$ membranous staining, was the most frequently reported in up to $20 \%$ of cases, whereas HER2 amplification and mutation represented $9 \%$ and $3 \%$ of cases, respectively. ${ }^{9}$ While the prevalence of HER2 mutation is near that of breast cancer $(2 \%)^{17}$ and gastric adenocarcinoma $(3 \%)^{18}$, prevalence of amplification is lower $\left(20-30 \%{ }^{19}\right.$ and up to $34 \%,{ }^{20}$ respectively). To assist in the detection of such targetable biomarkers, liquid biopsy (Guardant360 CDx) has recently been validated for use in lung cancer. In the NILE study, comprehensive cell-free DNA (cfDNA) analysis from patients with newly diagnosed metastatic NSCLC was compared to standard of care tissue genotyping. Use of cfDNA successfully identified guidelinerecommended biomarkers, including HER 2 alterations, at similar rates to tissue testing with a faster turnaround time. $^{21}$ As opposed to tissue biopsy, cfDNA analysis carries several advantages including easy sampling, mitigation of potential heterogeneity in intra-tumoral HER2 expression/amplification, and avoidance of biopsy complications. The ease of sampling has increased the frequency of detecting targetable mutations, including HER2.

\section{HER2-Targeted Therapy}

HER2-targeted agents have significantly improved the prognosis of HER2-positive breast cancer. As documented by Seah et al, the overall survival of HER2-positive metastatic breast cancer patients increased to 4.5 years. $^{22}$ NCCN guidelines have also incorporated the addition of HER2 inhibition in the first-line setting to HER2expressing gastric cancers. Unfortunately, such success has yet to be replicated in lung cancer, and there are currently no FDA-approved HER2-targeted therapies in this setting. The anti-HER2 monoclonal antibody trastuzumab was evaluated in combination with gemcitabine/cisplatin in treatment-naïve NSCLC patients with no evidence of improved clinical activity, ${ }^{23}$ as well as in combination with docetaxel in those who progressed on platinum-based therapy with disappointing results. ${ }^{24}$ A Phase II randomized clinical trial showed no difference in response rate or median survival between trastuzumab with docetaxel and trastuzumab with paclitaxel in previously untreated patients. ${ }^{25}$ More recently, however, the combination of trastuzumab and paclitaxel in EGFRmutated and HER2-expressing ( $\geq \mathrm{IHC} 1+$ ) NSCLC that progressed on first-line TKI therapy demonstrated acceptable tolerability with a promising objective response rate of $46 \%{ }^{26}$ Pertuzumab, a HER2 dimerization inhibitor that binds to a separate domain, initially showed antitumor activity in preclinical studies of NSCLC. ${ }^{27}$ Despite a promising Phase I clinical trial, ${ }^{28}$ two phase II trials of pertuzumab monotherapy in previously treated NSCLC patients showed no response. $^{29,30}$ The combination of pertuzumab and erlotinib initially showed a response rate of $20 \%$ in EGFR-mutated NSCLC in a Phase $1 \mathrm{~b}$ trial, ${ }^{31}$ but the combination was not pursued after unacceptable toxicity was demonstrated in a subsequent phase II trial. ${ }^{32}$

Trastuzumab Emtansine (T-DM1) was the first antibody-drug conjugate (ADC) tested in advanced HER2mutated NSCLC. A phase II trial compared the efficacy of T-DM1 by HER2 expression and showed a modest response rate of $20 \%$ in metastatic NSCLC expressing HER2 3+ by IHC, but the response was not seen in those with $2+$ IHC. Moreover, no PFS or OS advantage was observed in either cohort. ${ }^{33,34}$ Another phase II trial in HER2-positive NSCLC including IHC 3+, exon 20 mutated, and IHC $2+$ with positive FISH was terminated due to lack of efficacy of T-DM1. ${ }^{35}$ To date, only one trial 


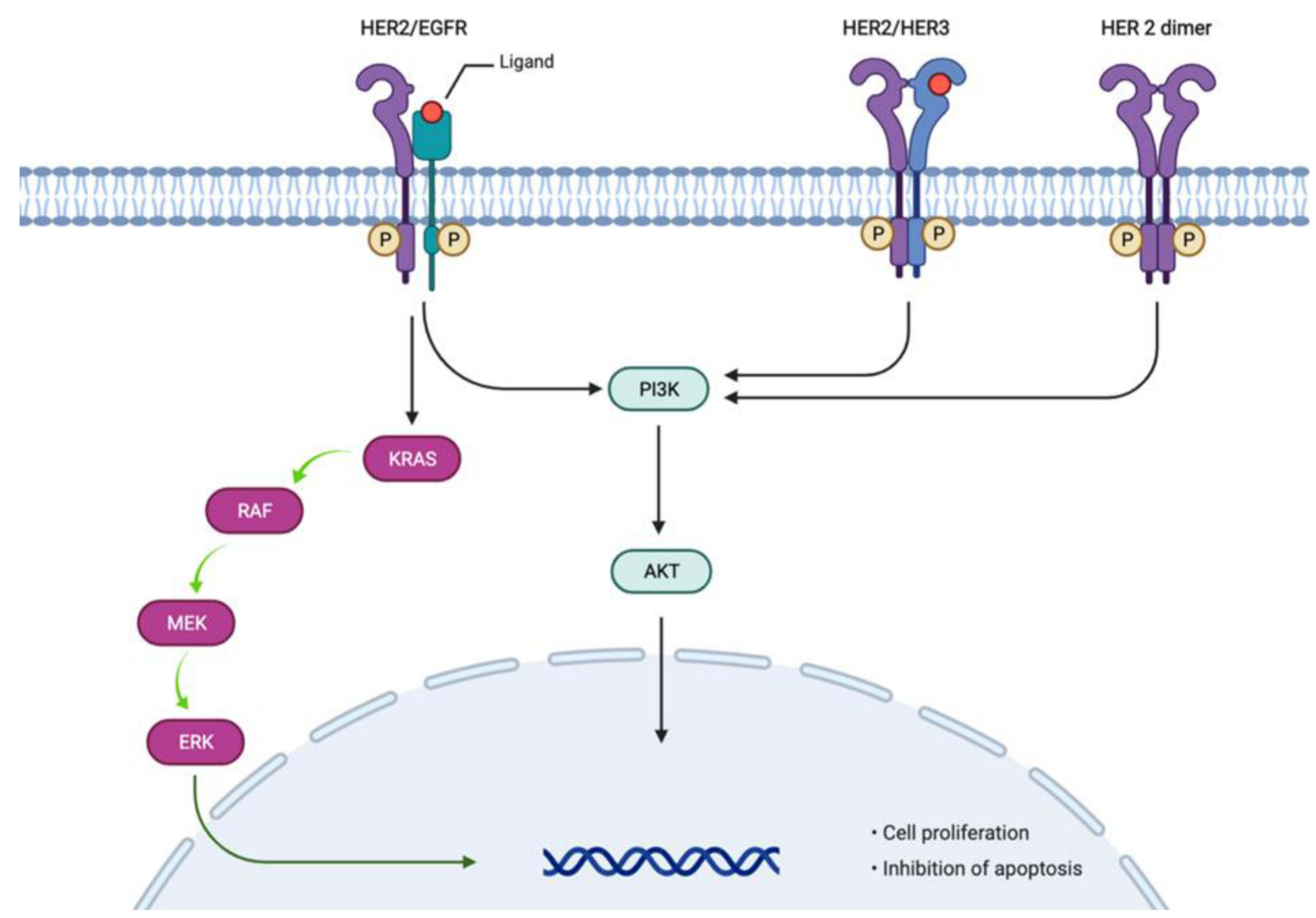

Figure I HER2 signaling pathway (Adapted from “HER2 Signaling Pathway”, by BioRender.com (202I). Retrieved from https://app.biorender.com/biorender-templates).

by Li et al demonstrated an encouraging median PFS of 5 months in this setting. ${ }^{36}$ Clinical efficacy was also demonstrated in HER2-amplified NSCLC. ${ }^{37}$

\section{Trastuzumab Deruxtecan}

Trastuzumab deruxtecan (T-DXd) was originally developed by Daiichi Sankyo (now being co-developed with AstraZeneca) as a novel antibody-drug conjugate (ADC) targeting HER2. T-DXd gained FDA approval in December 2019 for unresectable or metastatic HER2positive breast cancer after two or more prior lines of antiHER 2 therapy ${ }^{38}$. In the Phase II trial evaluating T-DXd in HER2-positive metastatic breast cancer patients who had received previous treatment with trastuzumab emtansine, the median duration of response to T-DXd was 14.8 months (95\% CI, 13.8 to 16.9 ) and the median duration of progression-free survival was 16.4 months $(95 \% \mathrm{CI}, 12.7$ to not reached) ${ }^{39}$ Subsequently, the FDA approved T-DXd (famtrastuzumab deruxtecan-nxki) in January 2021 for locally advanced or metastatic HER2 positive gastric or gastroesophageal junction (GEJ) adenocarcinoma based on the results of DESTINY-Gastric01. ${ }^{40}$ In this trial, T-DXd was superior to single-agent chemotherapy (irinotecan or paclitaxel) as a third (or later) line treatment for HER2-positive gastric and GEJ adenocarcinoma with a median OS of 12.5 months in the T-DXd arm compared to 8.4 months in the chemotherapy arm (HR 0.59; 95\% CI: 0.39, 0.88, $\mathrm{p}=0.0097)$ and ORR of $40.5 \%$ with T-DXd compared to $11.3 \%$ in those treated with chemotherapy. T-DXd was also evaluated in HER2positive refractory colon cancer patients (phase II DESTINYCRC01 trial) and showed promising results with ORR of $45 \%$ at a median follow-up of 27 weeks. $^{41}$ T-DXd is currently under investigation in numerous tumor types including biliary tract, urothelial bladder cancer, cervical cancer, endometrial cancer, ovarian cancer, pancreatic cancer (NCT04482309), and lung cancer. ${ }^{42}$

This review will focus on the role of T-DXd in lung cancer. We will discuss the structural characteristics, pharmacodynamics, and pharmacokinetics of T-DXd, and shed light on the preclinical and ongoing clinical trials of T-DXd along with the future directions in the management of HER2-positive lung cancer. 

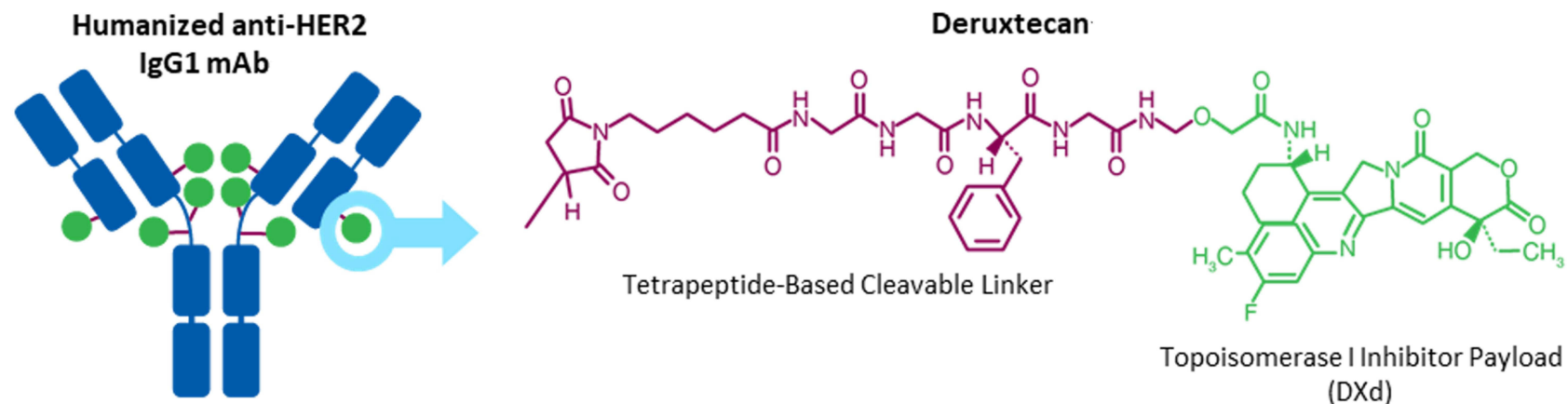

Topoisomerase I Inhibitor Payload (DXd)

Figure 2 Structure of Trastuzumab Deruxtecan.

Notes: Reproduced with permission from Egbert F. Smit, Kazuhiko Nakagawa, Misako Nagasaka, et al. Trastuzumab deruxtecan (T-DXd; DS-820I) in patients with HER2mutated metastatic non-small cell lung cancer (NSCLC): Interim results of DESTINY-Lung0I. J Clin Oncol. 2020;38(I5_suppl):9504. copyright 2020, Wolters Kluwer Health, Inc. $^{56}$

\section{Structural Characteristics of T-DXd}

Trastuzumab deruxtecan (previously called DS-8201a) is an antibody-drug conjugate (ADC) composed of three portions: an anti-HER2 antibody, a maleimide peptide linker, and a cytotoxic payload (DX-8951f) ${ }^{43,44}$ (Figure 2). The anti-HER2 antibody, MAAL-9001, is a humanized monoclonal immunoglobulin G1 with the same amino acid sequence as trastuzumab. Binding to HER2 positive tumor cells by MAAL-9001 leads to drug endocytosis. ${ }^{44}$ The tetrapeptide linker conjugates the cytotoxic load to the antibody. When circulating in the blood stream, the linker remains stable; however, once inside the cell, cleavage by lysosomal cathepsins releases the cytotoxic payload. Since cathepsins are upregulated in tumor cells, selective targeting of these cells occurs and limits systemic toxicity. ${ }^{45,46}$ DX-8951f, and its derivative MAAA-1181a (DXd), are topoisomerase I inhibitors of 10 -fold potency compared to the active metabolite of irinotecan (SN-38) in vitro. ${ }^{38}$ The payload-linker complex is connected to the antibody by cysteine-based residues. Drug-to-antibody ratio (DAR) is defined by the maximum number of payload molecules that are attached to the antibody. One of the key characteristics of T-DXd is a higher DAR ( $\sim 7$ to 8$)$ compared with other ADCs, enhancing antitumor activity. ${ }^{43}$ Additionally, the lipophilic nature of DXd allows for a significant bystander effect. ${ }^{47}$

\section{Pharmacodynamics}

By binding to HER2-positive tumor cells, the antigenADC complex undergoes endocytosis and the tetrapeptide linker is cleaved by lysosomal cathepsins, releasing the payload. DXd, in turn, inhibits DNA replication and induces apoptosis through binding of topoisomerase I-DNA complex (Figure 3). In in vitro pharmacologic studies, ${ }^{48}$ DXd inhibited DNA topoisomerase I with an IC50 of $0.31 \mu \mathrm{mol} / \mathrm{L}$ (vs 2.78 for SN-38). In addition, MAAL-9001 blocks Akt phosphorylation, leading to cell growth inhibition. Chk1 and Histone HAX2.X phosphorylation is also stimulated by DXd, resulting in DNA damage. Therefore, T-DXd combines the pharmacological activity of MAAL-9001, the antibody component, and MAAA-1181a, the cytotoxic part, by exhibiting HER2specific cell growth inhibition and antitumor activity, respectively. In a HER2-positive gastric cancer NCI-N87 model, ${ }^{48}$ tumor regression was observed with T-DXd but not with T-DM1, which was attributed to the difference in payload mechanism of action.

\section{Pharmacokinetics}

Pharmacokinetic profiles of T-DXd were studied in HER2positive tumor-bearing mice. ${ }^{49}$ Following IV administration of T-DXd, the pharmacokinetic profiles of the T-DXd and the total antibody (conjugated and unconjugated) were almost similar, indicating that the bio-linker is stable in circulation. This observation may be a significant factor in limiting systemic exposure and toxicity of DXd.

Biodistribution studies using radiolabeled T-DXd demonstrated tumor-specific distribution and long-term retention. DXd was the main catabolite released from T-DXd in tumors, with exposure levels at least five times higher than those in normal tissues and seven times higher than those achieved by a non-targeted control ADC. After IV administration of DXd, there was rapid clearance from the circulation (T1/2: $1.35 \mathrm{~h})$ and excretion was mainly through feces in its intact form. 


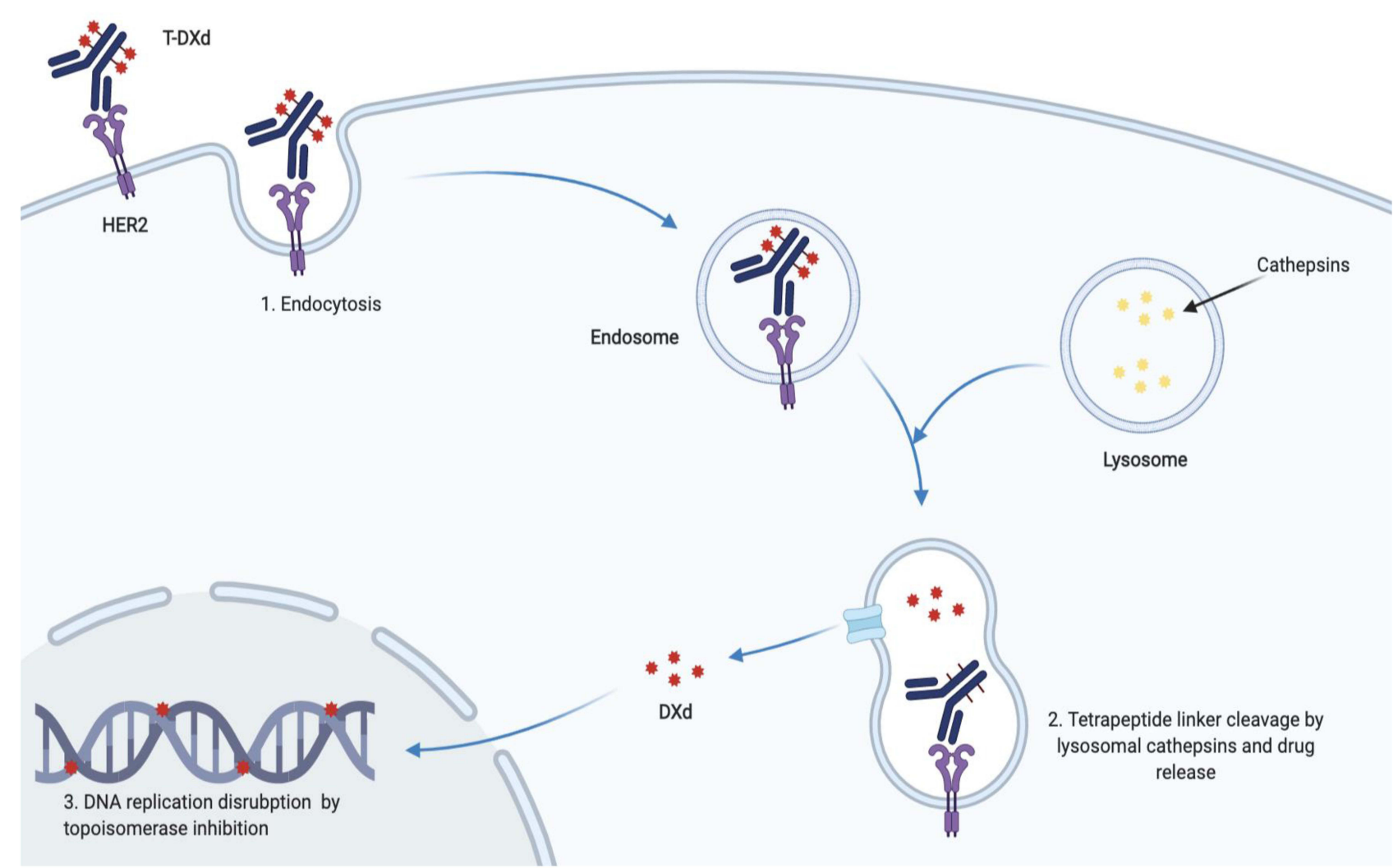

Figure 3 Trastuzumab Deruxtecan mechanism of action (Adapted from “Antibody-Drug Conjugate Drug Release”, by BioRender.com (202I). Retrieved from https://app. biorender.com/biorender-templates).

DXd is primarily metabolized by cytochrome P450 (CYP) 3A4 in vitro. Organic anion transporting peptide (OATP) was proposed to be responsible for payload uptake by the liver. ${ }^{50}$ Trastuzumab degradation is proposed to be similar to that of endogenous IgG. Clearance of T-DXd is approximately $0.42 \mathrm{~L} /$ day. ${ }^{38}$ Decreased clearance and prolonged half-life of T-DXd were associated with higher dosing, indicating a non-linear process. DXd did not exhibit inhibition or induction potential of CYP1A2 or CYP2B6. ${ }^{50}$ No antibodies against T-DXd were detected in the blood. The recommended dose based on clinical studies in metastatic breast and gastric cancers is $5.4 \mathrm{mg} /$ $\mathrm{kg}$ and $6.4 \mathrm{mg} / \mathrm{kg}$, respectively. ${ }^{51}$

\section{Preclinical Studies}

Antitumor efficacy of T-DXd was correlated with HER2 protein expression, not HER2 gene amplification. ${ }^{38}$ Activity was even detected in cell lines expressing low levels of HER $2 .{ }^{52}$ Due to the high membrane permeability of DXd, bystander killing by T-DXd was observed in vitro. Within the tumor tissue, HER2-negative cells that are adjacent to HER2-positive cells were affected. ${ }^{47,52}$ This finding, which has not been demonstrated in T-DM1, is clinically significant in tumors with HER2 heterogeneity. ${ }^{53}$ In addition, T-DM1 resistant xenograft models were sensitive to T-DXd. In N87-TDMR (T-DM1 resistant gastric cell line), efflux of the payload due to upregulation of cell pumps was suggested to be the mechanism of resistance to T-DM1. Thus, the activity of T-DXd was proposed to be either from low interaction between DXd and efflux pumps, or compensation for efflux activity by high DAR. ${ }^{54}$ Antitumor activity of T-DXd was even detected in low HER2 expression. ${ }^{48,52}$

\section{Clinical Trials}

Multiple investigational studies are being conducted in NSCLC either as monotherapy or in conjugation with other drug classes (Table 1).

\section{Phase I/II Trials}

T-DXd was investigated in a basket phase I study of multiple types of HER2-expressing or mutant solid tumors. ${ }^{55}$ Out of 18 patients with NSCLC, 11 harbored HER 2 mutations, with the most common being exon 20 
Table I Clinical Trials of Trastuzumab Deruxtecan in Non-Small Cell Lung Cancer (NSCLC)

\begin{tabular}{|c|c|c|c|c|}
\hline NCT & Title & Phase & Combination Therapy & Status \\
\hline 04644237 & $\begin{array}{c}\text { A Phase 2, Multicenter, Randomized Study of Trastuzumab Deruxtecan in } \\
\text { Subjects with HER2-mutated Metastatic Non-small Cell Lung Cancer (NSCLC) } \\
\text { (DESTINY- LUNG02) }\end{array}$ & II & N/A & Recruiting \\
\hline 03505710 & $\begin{array}{c}\text { A Phase 2, Multicenter, Open-Label, 2-Cohort Study of Trastuzumab } \\
\text { Deruxtecan (DS-820Ia), an Anti-HER2 Antibody Drug Conjugate (ADC), for } \\
\text { HER2-Over-Expressing or -Mutated, Unresectable and/or Metastatic Non-Small } \\
\text { Cell Lung Cancer (NSCLC) (DESTINY- LUNG0I) }\end{array}$ & II & N/A & $\begin{array}{l}\text { Active, not } \\
\text { recruiting }\end{array}$ \\
\hline 04042701 & $\begin{array}{l}\text { A Phase Ib, Multicenter, Two-Part, Open-Label Study of Trastuzumab } \\
\text { Deruxtecan (DS-820 Ia), An Anti-Human Epidermal Growth Factor Receptor-2 } \\
\text { (HER2)-Antibody Drug Conjugate (ADC), In Combination with Pembrolizumab, } \\
\text { An Anti-PD-I Antibody, In Subjects with Locally Advanced/Metastatic Breast or } \\
\text { Non-Small Cell Lung Cancer (NSCLC) }\end{array}$ & $\mathrm{lb}$ & Pembrolizumab & Recruiting \\
\hline 04686305 & $\begin{array}{c}\text { A Phase lb Multicenter, Open-label Dose-escalation Study to Evaluate the Safety } \\
\text { and Tolerability of Trastuzumab Deruxtecan (T-DXd) and Durvalumab in } \\
\text { Combination with Cisplatin, Carboplatin or Pemetrexed in First-line Treatment } \\
\text { of Patients with Advanced or Metastatic Non-squamous Non-small Cell Lung } \\
\text { Cancer (NSCLC) and Human Epidermal Growth Factor Receptor } 2 \\
\text { Overexpression (HER2+) (DESTINY- Lung03) }\end{array}$ & $\mathrm{lb}$ & $\begin{array}{l}\text { - Durvalumab } \\
\text { - Durvalumab + } \\
\text { Cisplatin } \\
\text { - Durvalumab + } \\
\text { Carboplatin } \\
\text { - Durvalumab + } \\
\text { Pemetrexed }\end{array}$ & $\begin{array}{l}\text { Active, not } \\
\text { recruiting }\end{array}$ \\
\hline 03334617 & $\begin{array}{l}\text { An Open-Label, Multi-Drug, Biomarker-Directed, Multi-Centre Phase II } \\
\text { Umbrella Study in Patients with Non-Small Cell Lung Cancer, Who Progressed } \\
\text { on an Anti-PD-I/PD-LI Containing Therapy (HUDSON) }\end{array}$ & II & Durvalumab & Recruiting \\
\hline
\end{tabular}

insertions. IHC score for HER2 expression was $1+$ in 8 patients, $2+$ in one, and $3+$ in two patients. Five had an IHC score of 0 and two patients lacked evaluation of HER2 expression. Only five patients had been previously treated with HER2-targeted therapy. T-DXd was dosed at $6.4 \mathrm{mg} / \mathrm{kg}$. Confirmed objective response rate (ORR) for all tumor types was $55.6 \%$, and median PFS was 11.3 months (95\% CI, 7.2-14.3 months). However, response to treatment in HER2-mutant NSCLC was more evident with ORR and disease control rate (DCR) of $72.7 \%$ and $90.9 \%$, respectively. Treatment-related adverse events were reported in two patients only (11.1\%). It was concluded that T-DXd was clinically active and well tolerated across multiple types of tumors, particularly HER2-mutant NSCLC. ${ }^{55}$

The DESTINY-Lung01 trial evaluated T-DXd at a dose of $6.4 \mathrm{mg} / \mathrm{kg}$ in two separate cohorts of non-squamous NSCLC: HER2-mutated and HER2-overexpressed (IHC 2 + and IHC 3+). Patients with central nervous system metastases were eligible, unless untreated, symptomatic, or requiring corticosteroids or anticonvulsants. From May 21, 2018 until data cutoff on November 25th, 2019,
42 patients with HER2-mutant NSCLC were enrolled and interim data for this group were presented at the ASCO 2020 meeting. The median number of prior lines of therapy was 2 and included platinum-based chemotherapy, as well as PD-1 and PD-L1 inhibitors. Twenty-six patients had confirmed response $(61.9 \%)$ with DCR of $90.5 \%$ (95\% CI, 77.4-97.3\%) and a median PFS of 14 months. In addition, median duration of response (DOR) was not achieved at the time of data cutoff. ${ }^{56}$ Given these results, T-DXd was granted a breakthrough therapy designation in May 2020 for patients with metastatic HER2-mutated NSCLC who progressed on or after platinum-based chemotherapy and is currently listed on the NCCN guidelines. $^{57}$

Updated results for the HER2-overexpressing cohort were presented at IASLC WCLC in January 2021. ORR and DCR from the 49 enrolled patients were lower than that of the HER2-mutant cohort $(24.5 \%$ and $69.1 \%$, respectively) with a median duration of response of 6 months. Median PFS was 5.4 months (Table 2). Ultimately, T-DXd, at $6.4 \mathrm{mg} / \mathrm{kg}$, demonstrated antitumor activity in this cohort. ${ }^{58}$ 
Table 2 Comparison of DESTINY-Lung0I Trial Results Between HER-Mutant and HER2-Overexpressing Cohorts

\begin{tabular}{|c|c|c|}
\hline \multicolumn{3}{|c|}{ DESTINY-Lung0 I } \\
\hline & HER Mutation & HER2 Overexpression \\
\hline Patients (n) & 42 & 49 \\
\hline Objective response rate & $n=26(61.9 \%)$ & $\mathrm{n}=2(24.5 \%)$ \\
\hline Complete response & $\mathrm{n}=\mathrm{l}$ & $\mathrm{n}=\mathrm{I}$ \\
\hline Partial response & $\mathrm{n}=25$ & $\mathrm{n}=\mathrm{II}$ \\
\hline Duration of response & Not reached & 6 months \\
\hline Disease control rate & $90.5 \%$ & $69.1 \%$ \\
\hline Progression-free survival & 14 months & 5.4 months \\
\hline Dose interruption & $\mathrm{n}=25(59.5 \%)$ & $n=26(53.1 \%)$ \\
\hline Dose reduction & $\mathrm{n}=16(38.1 \%)$ & $\mathrm{n}=17(34.7 \%)$ \\
\hline Dose discontinuation & $\mathrm{n}=10(23.8 \%)$ & $\mathrm{n}=1 \mathrm{I}(22.4 \%)$ \\
\hline Treatment emergent adverse events & $n=42(100 \%)$ & $\mathrm{n}=49(100 \%)$ \\
\hline Nausea & $\mathrm{n}=32(76.2 \%)$ & $n=29(59.2 \%)$ \\
\hline Alopecia & $n=20(47.6 \%)$ & - \\
\hline Anemia & $\mathrm{n}=18(42.9 \%)$ & - \\
\hline Decreased appetite & $\mathrm{n}=18(42.9 \%)$ & $n=19(38.8 \%)$ \\
\hline Decreased neutrophil count & $\mathrm{n}=18(42.9 \%)$ & - \\
\hline Fatigue & - & $\mathrm{n}=16(32.7 \%)$ \\
\hline Grade 3 adverse events & $n=27(64.3 \%)$ & $n=36(73.5 \%)$ \\
\hline Decreased neutrophil count & $\mathrm{n}=\mathrm{II}(26.2 \%)$ & $n=10(20.4 \%)$ \\
\hline Anemia & $n=7(16.7 \%)$ & - \\
\hline Fatigue & - & $n=5(10.2 \%)$ \\
\hline
\end{tabular}

\section{Safety, Tolerability, and Toxicity Profile}

In the DESTINY-Lung01 study, interim data from the HER2-mutant group reported grade 3 treatment-related adverse events in over half of the participants $(64.3 \%)$, including neutropenia and anemia. Out of the 42 enrolled patients, development of Interstitial Lung Disease (ILD) was demonstrated in 5 patients (All grade 2). Due to treatment-emergent adverse events (TEAEs), 10 patients had to discontinue treatment $(23.8 \%)$ and 16 received dose-reduction (38.1\%), while 25 had dose-interruptions $(59.5 \%){ }^{56}$ Updated safety results in 49 patients with HER2-overexpression demonstrated nausea, decreased appetite, and fatigue as the most common TEAE. Grade $\geq 3$ TEAEs were reported in $73.5 \%$ of patients and included neutropenia and fatigue. T-DXd dose had to be reduced or interrupted in 17 and 26 patients, respectively. Compared to the HER2-mutant cohort, the incidence of ILD was slightly higher (16.3\% vs $11.5 \%)$, and grade 4 severity was reported in 3 patients (Table 3). Despite the demonstrated tolerability, ILD remains a concerning and serious adverse event. ${ }^{58}$

In a phase I dose-escalation study, 24 patients with breast, gastric, and gastroesophageal cancers were treated with different doses of T-DXd. Gastrointestinal and hematological events were reported. Three patients experienced serious adverse events: intestinal perforation, febrile neutropenia, and cholangitis. Three patients had to discontinue treatment due to drug-induced toxicity, including thrombocytopenia and pneumonitis. Higher doses were more heavily associated with high-grade adverse events. Unlike trastuzumab, cardiac toxicity was not observed across all cohorts. ${ }^{45}$

In a recent trial in patients with low HER2-expressing breast cancer, T-DXd was administered at $5.4 \mathrm{mg} / \mathrm{kg}$ or $6.4 \mathrm{mg} / \mathrm{kg}$. Eleven out of 54 participants (20.4\%) discontinued therapy due to adverse events, most commonly pneumonitis and ILD. Grade III toxicity, in addition to 
Table 3 ILD Incidence Rate and Grading Among DESTINY Trials

\begin{tabular}{|c|c|c|c|c|}
\hline & DESTINY-Breast0I & DESTINY-Gastric0 I & \multicolumn{2}{|c|}{ DESTINY-Lung0I } \\
\hline & & & HER2 Mutation & $\begin{array}{c}\text { HER2 } \\
\text { Overexpression }\end{array}$ \\
\hline Number of treated patients & 184 & 125 & 42 & 49 \\
\hline T-DXd dose & $5.4 \mathrm{mg} / \mathrm{kg}$ & $6.4 \mathrm{mg} / \mathrm{kg}$ & $6.4 \mathrm{mg} / \mathrm{kg}$ & $6.4 \mathrm{mg} / \mathrm{kg}$ \\
\hline Number of patients with ILD & $25(13.6 \%)$ & $12(10 \%)$ & 5 (11.5\%) & $8(16.3 \%)$ \\
\hline \multicolumn{5}{|c|}{ ILD Grading } \\
\hline I & $20(80 \%)$ & $9(75 \%)$ & & $2(25 \%)$ \\
\hline II & & & $5(100 \%)$ & $3(37.5 \%)$ \\
\hline III & I (4\%) & $2(16.7 \%)$ & & \\
\hline IV & & I (8.3\%) & & \\
\hline V & $4(16 \%)$ & & & $3(3.75 \%)$ \\
\hline
\end{tabular}

Notes: Pneumonitis grading: Grade I: asymptomatic; clinical or diagnostic observations only; intervention not indicated; Grade 2: symptomatic; medical intervention indicated; limiting instrumental ADL; Grade 3: severe symptoms; limiting self-care ADL*; oxygen indicated; Grade 4: life-threatening respiratory compromise; urgent intervention indicated (eg, tracheostomy or intubation); Grade 5: death. Shading refers to no ILD reported at the specific grade.

treatment discontinuation and interruption, were more frequently reported in the high-dose group. Drug-induced ILD occurred in eight patients $(14.8 \%)$, all in the $6.4 \mathrm{mg} /$ $\mathrm{kg}$ cohort, leading to death in three patients despite corticosteroid therapy. ${ }^{59}$

In the DESTINY-Breast01 trial, T-DXd was evaluated at a dose of $5.4 \mathrm{mg} / \mathrm{kg}$ in 184 patients. Leukopenia, anemia, fatigue, and nausea were the most commonly observed grade $\geq 3$ toxicities. Febrile neutropenia was witnessed in three patients. Grade 2 and 3 systolic dysfunction was reported in 3 asymptomatic patients, whose ejection fraction recovered after holding therapy. Nine patients $(4.9 \%)$ had QT interval prolongation. ILD and pneumonitis were the main reasons for treatment discontinuation. Treatment-induced ILD was reported in 25 patients (13.6\%), predominantly of low-grade severity. Median time of onset was 193 days with median time to recovery of 34 days. Recovery was demonstrated in seven patients $(28 \%))^{39}$ Of the 20 patients who were reported to have ILD of grade 2 or higher, 13 received glucocorticoids and 7 were hospitalized with four deaths $(2.2 \%$ of the patients) attributable to interstitial lung disease. ${ }^{39}$

Hematologic toxicity was similar in DESTINYGastric01, where 125 patients received T-DXd at a dose of $6.4 \mathrm{mg} / \mathrm{kg}$. Six patients developed febrile neutropenia. Treatment-related systolic dysfunction was not demonstrated. ILD or pneumonitis were reported in 12 participants $(10 \%)$, none of grade 5 severity; median time until the date of onset was 84.5 days. Eight cases had recovered or were recovering at the time of analysis. ${ }^{40}$

\section{Ongoing T-DXd Trials}

Results of the phase I DESTINY-Lung01 trial achieved breakthrough designation status for the use of T-DXd in metastatic HER2-mutated NSCLC progressing on platinum-based therapy, and full FDA approval is anticipated with the completion of the subsequent DESTINYLung02 trial. T-DXd has been approved at a dose of $5.4 \mathrm{mg} / \mathrm{kg}$ for metastatic HER2-positive breast cancer and $6.4 \mathrm{mg} / \mathrm{kg}$ for gastric cancer. DESTINY-Lung02 (NCT0464437), a randomized phase II study comparing the two dose levels of 5.4 or $6.4 \mathrm{mg} / \mathrm{kg}$, has started enrolling in March 2021 (Figure 4). Patients who have received at least one line of platinum-based therapy are eligible with the primary endpoint being objective response rates.

The optimal sequencing of T-DXd and chemoimmunotherapy in HER2-mutated NSCLC will likely be addressed in future trials. Few trials are looking into whether combining T-DXd with immunotherapy or chemoimmunotherapy holds a therapeutic advantage. The combination of T-DXd and pembrolizumab will be investigated in a phase Ib study in patients with locally advanced and metastatic HER2-positive breast and 


\section{DESTINY-Lung02}

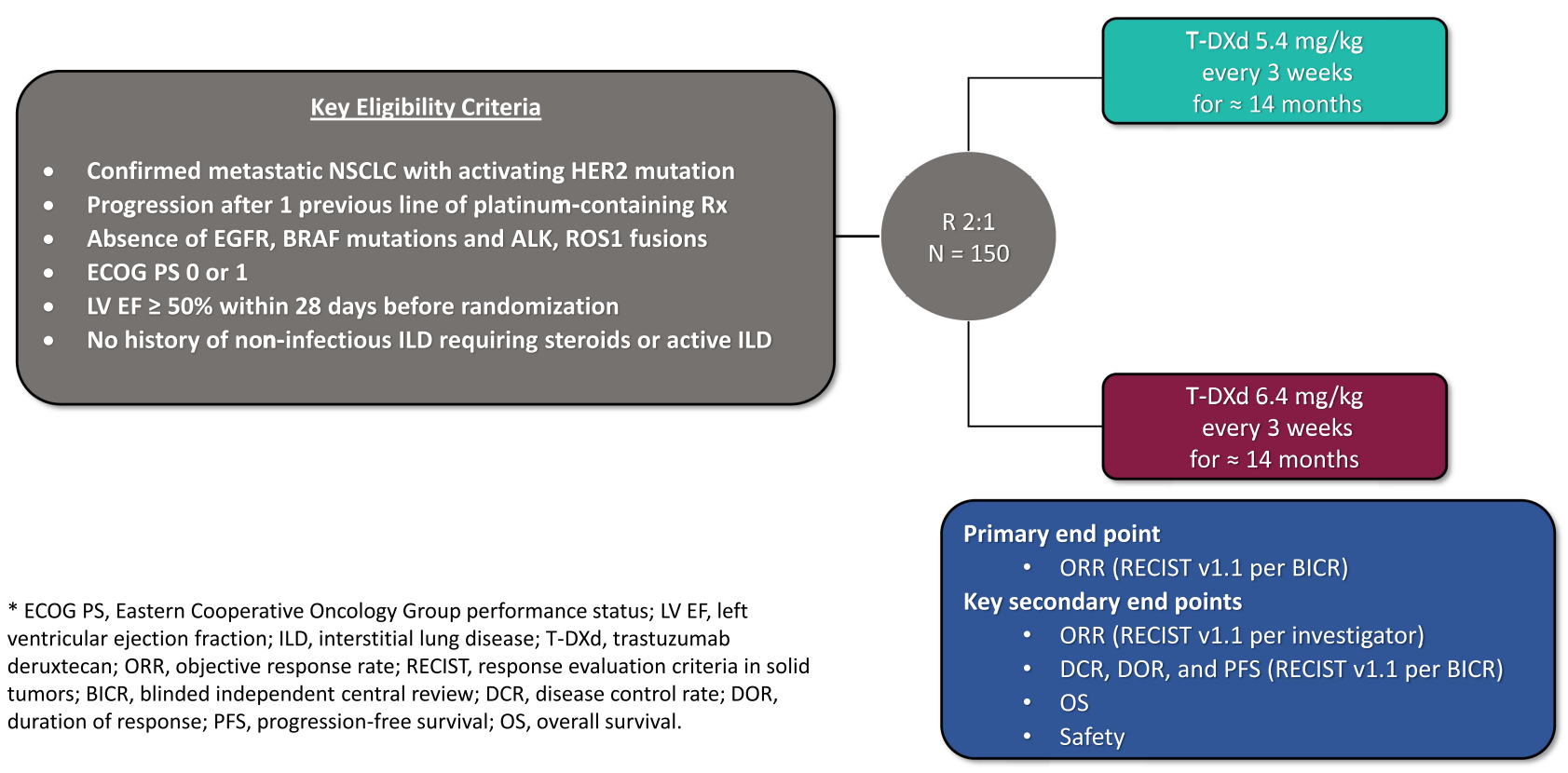

Figure 4 Schema for DESTINY-Lung02. Additional details can be found at: https://clinicaltrials.gov/ct2/show/NCT04644237.

Abbreviations: *ECOG PS, Eastern Cooperative Oncology Group performance status; LV EF, left ventricular ejection fraction; ILD, interstitial lung disease; T-DXd, trastuzumab deruxtecan; ORR, objective response rate; RECIST, response evaluation criteria in solid tumors; BICR, blinded independent central review; DCR, disease control rate; DOR, duration of response; PFS, progression-free survival; OS, overall survival.

NSCLC who did not receive prior PD-1, PD-L1 or HER2targeted therapy. In part 1 (dose-escalation phase), T-DXd will be administered at a dose of 3.2 or $5.4 \mathrm{mg} / \mathrm{kg}$. The recommended dose for part 2 (expansion phase) will be determined in the dose-escalation part. HER2-mutant or HER2-expressing (IHC $\geq 1+$ ) NSCLC patients will be enrolled. The first patient enrolled on February 10th, 2020. Dose-limiting toxicity and confirmed ORR are the primary outcomes in dose-escalation and expansion parts, respectively. $^{60}$ Similarly, the HUDSON trial (NCT03334617), an umbrella phase II trial, is ongoing to evaluate the combination of durvalumab with other classes of drugs, including T-DXd. Multiple arms of treatment are under evaluation, including NSCLC patients who have progressed to PD-1/PD-L1 inhibition. T-DXd will be administered at a dose of $5.4 \mathrm{mg} / \mathrm{kg}$ with primary endpoint being objective response rate. DESTINY-Lung03, a phase $1 \mathrm{~b}$ trial, evaluating the safety of T-DXd combined with immuno- and cytotoxic therapy is also underway. Patients with treatment-naïve HER2-overexpressing (IHC 3+ or 2 +) NSCLC will be included. Initially, at least six patients will receive T-DXd plus durvalumab for safe run-in. This is followed by dose-escalation, where patients will be treated with T-DXd plus durvalumab and cytotoxic therapy (either cisplatin, carboplatin, or pemetrexed). Optional dose-expansion will be conducted depending on data from the dose-escalation phase. In addition to safety evaluation, determination of an appropriate phase II dose is the primary endpoint. ${ }^{61}$ The safety profile of combination therapy, particularly the potential for developing pneumonitis, will be critical to the fate of these combinations.

While the response rate of T-DXd in HER2overexpressing NSCLC was less impressive (ORR $24.5 \%$ ), the patients treated had a median of 3 previous lines of therapy. After progression to platinum- and checkpoint inhibitor-based therapies, the current options for adenocarcinoma of the lung consist of single-agent chemotherapy (docetaxel, pemetrexed, gemcitabine). The addition of ramucirumab to docetaxel only improves survival by 1.4 months. ${ }^{62}$ As such, T-DXd might cement a place as an attractive option in subsequent lines of therapy. The efficacy and tolerability of T-DXd at a dose of $5.4 \mathrm{mg} / \mathrm{kg}$ in HER2-amplified solid tumors is under evaluation in the phase II basket (HERALD) trial. Guardant360 cfDNA analysis will be utilized to identify HER 2 amplification. In addition, predictive biomarkers are 
evaluated using serial collection of tumor tissue and cfDNA. $^{63}$

\section{Novel HER2 TKIs Under Investigation}

Besides T-DXd, targeted HER2 treatment of NSCLC is currently under investigation utilizing 3 different tyrosine kinase inhibitors. Poziotinib showed high potency in preclinical studies of EGFR exon 20 mutation. ${ }^{64}$ Poziotinib was investigated in a phase II clinical trial in patients with previously treated, EGFR-mutated or exon 20 HER2-mutant NSCLC. Although the initial clinical activity was demonstrated with ORR and DCR of $50 \%$ and $83 \%$, respectively, in the HER 2 exon 20 mutation cohort, ${ }^{65}$ more mature data presented at AACR 2020 showed a disappointing RR of $27.8 \% .{ }^{66}$ Preclinical studies have demonstrated antitumor activity of poziotinib in combination with T-DM1 in NSCLC with exon $20 \mathrm{HER} 2$ mutation. By upregulating mutant HER2 in tumor cells, poziotinib enhances the clinical activity of T-DM1, leading to complete regression. ${ }^{67}$ Clinical trials of this combination could be considered. Tarloxotinib is a hypoxia-activated prodrug of a pan-ErbB kinase inhibitor that releases a potent irreversible active metabolite (tarloxotinib-E) to preferentially target tumor tissues. Tarloxotinib has demonstrated preclinical efficacy in EGFR exon 20 and HER2-mutant NSCLC, as well as other oncogenic alterations in the ERBB gene family such as NRG1 fusions. First reports from the Rain-701 trial showed a PR of $22 \%(2 / 9)$, SD of $44 \%$ (4/9), and PD of $33 \%(3 / 9) .{ }^{68}$ Mobocertinib is a first-in-class TKI designed to target EGFR and HER2 exon 20 insertions. While results for $E G F R$ exon 20 insertions from the EXCLAIM trial were reported at WCLC 2020, accrual for HER2 exon 20 insertions has closed (personal communication).

\section{Conclusion}

T-DXd holds significant promise in HER2-mutated NSCLC and, to a lesser-degree, in HER2-overexpressing NSCLC. The optimal strategy of using T-DXd in NSCLC is not yet well established. To this end, combination therapy utilizing T-DXd with checkpoint inhibitors and chemoimmunotherapy is under current investigation. Despite targeting HER2 mutated lung cells, pneumonitis and ILD remain a serious challenge. The identification of patients likely to develop this adverse event requires further investigation.

\section{Funding}

The authors have not received any funding for this study and declare no direct conflict of interest.

\section{Disclosure}

Dr. Al Hallak is on the speaker's bureau for Ipsen and is a consultant for HalioDx. Dr. Sukari serves on the advisory board for Merck and Eisai. He has received study funding from Eisai. Funding. Dr. Nagasaka serves on the advisory board for AstraZeneca, Caris Life Sciences, Daiichi-Sankyo, Takeda, Novartis, EMD Serono and has received study funding from Tempus. Dr. Nagasaka has been awarded the 2020 Karmanos Cancer Institute Cancer Immunology and Immunotherapy Pilot Award (P30 CA022453). All other authors have no potential conflict of interest to declare.

\section{References}

1. King C, Kraus M, Aaronson S. Amplification of a novel v-erbBrelated gene in a human mammary carcinoma. Science. 1985;229 (4717):974-976. doi:10.1126/science.2992089

2. Klapper LN, Glathe S, Vaisman N, et al. The ErbB-2/HER2 oncoprotein of human carcinomas may function solely as a shared coreceptor for multiple stroma-derived growth factors. Proc Natl Acad Sci. 1999;96(9):4995-5000.

3. Giatromanolaki A, Koukourakis Mi, Simopoulos C, et al. c-erbB-2 related aggressiveness in breast cancer is hypoxia inducible factor- $1 \alpha$ dependent. Clin Cancer Res. 2004;10(23):7972-7977.

4. Gassmann M, Casagranda F, Orioli D, et al. Aberrant neural and cardiac development in mice lacking the ErbB4 neuregulin receptor. Nature. 1995;378(6555):390-394.

5. Threadgill D, Dlugosz A, Hansen L, et al. Targeted disruption of mouse EGF receptor: effect of genetic background on mutant phenotype. Science. 1995;269(5221):230-234.

6. Kerbel RS, Viloria-Petit A, Klement G, Rak J. 'Accidental' antiangiogenic drugs. Eur J Cancer. 2000;36(10):1248-1257.

7. O-charoenrat P, Rhys-Evans P, Court WJ, Box GM, Eccles SA. Differential modulation of proliferation, matrix metalloproteinase expression and invasion of human head and neck squamous carcinoma cells by c-erbB ligands. Clin Exp Metastasis. 1999;17 (7):631-639.

8. Ménard S, Pupa SM, Campiglio M, Tagliabue E. Biologic and therapeutic role of HER2 in cancer. Oncogene. 2003;22(42):6570-6578.

9. Landi L, Cappuzzo F. HER2 and lung cancer. Expert Rev Anticancer Ther. 2013;13(10):1219-1228.

10. Slamon D, Godolphin W, Jones L, et al. Studies of the HER-2/neu proto-oncogene in human breast and ovarian cancer. Science. 1989;244(4905):707-712. doi:10.1126/science. 2470152

11. Leonetti A, Sharma S, Minari R, Perego P, Giovannetti E, Tiseo M. Resistance mechanisms to osimertinib in EGFR-mutated non-small cell lung cancer. Br J Cancer. 2019;121(9):725-737.

12. Ramalingam SS, Cheng Y, Zhou C, et al. Mechanisms of acquired resistance to first-line osimertinib: preliminary data from the Phase III FLAURA study. Ann Oncol. 2018;29:vii740.

13. Papadimitrakopoulou VA, Wu Y-L, Han J-Y, et al. Analysis of resistance mechanisms to osimertinib in patients with EGFR T790M advanced NSCLC from the AURA3 study. Ann Oncol. 2018;29: vii740. 
14. Pallis AG, Syrigos KN. Lung cancer in never smokers: disease characteristics and risk factors. Crit Rev Oncol Hematol. 2013;88 (3):494-503.

15. Mar N, Vredenburgh JJ, Wasser JS. Targeting HER2 in the treatment of non-small cell lung cancer. Lung Cancer. 2015;87(3):220-225.

16. Hotta K, Yanai H, Ohashi K, et al. Pilot evaluation of a HER2 testing in non-small-cell lung cancer. J Clin Pathol. 2020;73(6):353-357.

17. Ding Q, Chen H, Lim B, et al. HER2 somatic mutation analysis in breast cancer: correlation with clinicopathological features. Hum Pathol. 2019;92:32-38.

18. Lengyel CG, Habeeb B, Khan SZ, et al. Role of HER-2 in gastrointestinal tumours beyond gastric cancer: a tool for precision medicine. Gastrointestinal Disord. 2020;3(1):1-22.

19. Yi Z, Rong G, Guan Y, et al. Molecular landscape and efficacy of HER2-targeted therapy in patients with HER2-mutated metastatic breast cancer. NPJ Breast Cancer. 2020;6(1):59-undefined.

20. Kanayama K, Imai H, Usugi E, Shiraishi T, Hirokawa YS, Watanabe M. Association of HER2 gene amplification and tumor progression in early gastric cancer. Virchows Archiv. 2018;473 (5):559-565.

21. Leighl NB, Page RD, Raymond VM, et al. Clinical utility of comprehensive cell-free DNA analysis to identify genomic biomarkers in patients with newly diagnosed metastatic non-small cell lung cancer. Clin Cancer Res. 2019;25(15):4691-4700.

22. Seah DSE, Luis IV, Macrae E, et al. Use and duration of chemotherapy in patients with metastatic breast cancer according to tumor subtype and line of therapy. J Natl Compr Cancer Net. 2014;12 (1):71-80.

23. Gatzemeier U, Groth G, Butts C, et al. Randomized phase II trial of gemcitabine-cisplatin with or without trastuzumab in HER2-positive non-small-cell lung cancer. Ann Oncol. 2004;15(1):19-27.

24. Lara PN, Laptalo L, Longmate J, et al. Trastuzumab plus docetaxel in HER2/neu-positive non-small-cell lung cancer: a California cancer consortium screening and Phase II trial. Clin Lung Cancer. 2004;5 (4):231-236.

25. Krug LM, Miller VA, Patel J, et al. Randomized phase II study of weekly docetaxel plus trastuzumab versus weekly paclitaxel plus trastuzumab in patients with previously untreated advanced nonsmall cell lung carcinoma. Cancer. 2005;104(10):2149-2155.

26. de Langen AJ, Jebbink M, Hashemi SMS, et al. Trastuzumab and paclitaxel in patients with EGFR mutated NSCLC that express HER2 after progression on EGFR TKI treatment. Br J Cancer. 2018;119 (5):558-564.

27. Barthélémy P, Leblanc J, Goldbarg V, Wendling F, Kurtz J-E. Pertuzumab: development beyond breast cancer. Anticancer Res. 2014;34:1483-1491.

28. Agus DB, Gordon MS, Taylor C, et al. Phase I clinical study of pertuzumab, a novel HER dimerization inhibitor, in patients with advanced cancer. J Clin Oncol. 2005;23(11):2534-2543.

29. Herbst RS, Davies AM, Natale RB, et al. Efficacy and safety of single-agent pertuzumab, a human epidermal receptor dimerization inhibitor, in patients with non-small cell lung cancer. Clin Cancer Res. 2007;13(20):6175-6181.

30. Johnson BE, Jänne PA. Rationale for a Phase II trial of pertuzumab, a HER-2 dimerization inhibitor, in patients with non-small cell lung cancer: fig. 1. Clin Cancer Res. 2006;12(14):4436s-4440s

31. Felip E, Ranson M, Cedrés S, et al. A Phase IB, dose-finding study of erlotinib in combination with a fixed dose of pertuzumab in patients with advanced non-small-cell lung cancer. Clin Lung Cancer. 2012;13(6):432-441.

32. Hughes B, Mileshkin L, Townley P, et al. Pertuzumab and erlotinib in patients with relapsed non-small cell lung cancer: a Phase II study using 18F-fluorodeoxyglucose positron emission tomography/computed tomography imaging. Oncologist. 2014;19(2):175-176.
33. Stinchcombe T, Stahel RA, Bubendorf L, et al. Efficacy, safety, and biomarker results of trastuzumab emtansine (T-DM1) in patients (pts) with previously treated HER2-overexpressing locally advanced or metastatic non-small cell lung cancer (mNSCLC). J Clin Oncol. 2017;35(15_suppl):8509.

34. Peters S, Stahel R, Bubendorf L, et al. Trastuzumab Emtansine (T-DM1) in patients with previously treated HER2-overexpressing metastatic non-small cell lung cancer: efficacy, safety, and biomarkers. Clin Cancer Res. 2019;25(1):64-72.

35. Hotta K, Aoe K, Kozuki T, et al. A Phase II study of trastuzumab emtansine in HER2-positive non-small cell lung cancer. $J$ Thor Oncol. 2018;13(2):273-279.

36. Li BT, Shen R, Buonocore D, et al. Ado-trastuzumab emtansine for patients with HER2 -mutant lung cancers: results from a Phase II basket trial. J Clin Oncol. 2018;36(24):2532-2537.

37. Li BT, Michelini F, Misale S, et al. HER2-mediated internalization of cytotoxic agents in ERBB2 amplified or mutant lung cancers. Cancer Discov. 2020;10(5):674-687. doi:10.1158/2159-8290.CD-20-0215

38. Keam SJ. Trastuzumab deruxtecan: first approval. Drugs. 2020;80 (5):501-508.

39. Modi S, Saura C, Yamashita T, et al. Trastuzumab deruxtecan in previously treated HER2-positive breast cancer. $N$ Eng $\mathrm{J} \mathrm{Med.}$ 2020;382(7):610-621.

40. Shitara K, Bang Y-J, Iwasa S, et al. Trastuzumab deruxtecan in previously treated HER2-positive gastric cancer. $N$ Eng J Med. 2020;382(25):2419-2430.

41. Siena S, Di Bartolomeo M, Raghav K, et al. Trastuzumab deruxtecan (DS-8201) in patients with HER2-expressing metastatic colorectal cancer (DESTINY-CRC01): a multicentre, open-label, Phase 2 trial. Lancet Oncol. 2021;22(6):779-789.

42. Indini A, Rijavec E, Grossi F. Trastuzumab deruxtecan: changing the destiny of HER2 expressing solid tumors. Int J Mol Sci. 2021;22(9):4774.

43. Andrikopoulou A, Zografos E, Liontos M, Koutsoukos K, Dimopoulos M-A, Zagouri F. Trastuzumab deruxtecan (DS-8201a): the latest research and advances in breast cancer. Clin Breast Cancer. 2020.

44. Linehan AS, Fitzpatrick OM, Morris PG. Profile of Trastuzumab deruxtecan in the management of patients with HER2-positive unresectable or metastatic breast cancer: an evidence-based review. Breast Cancer. 2021;13:151-159.

45. Doi T, Shitara K, Naito Y, et al. Safety, pharmacokinetics, and antitumour activity of trastuzumab deruxtecan (DS-8201), a HER2targeting antibody-drug conjugate, in patients with advanced breast and gastric or gastro-oesophageal tumours: a phase 1 dose-escalation study. Lancet Oncol. 2017;18(11):1512-1522.

46. Tamura K, Tsurutani J, Takahashi S, et al. Trastuzumab deruxtecan (DS-8201a) in patients with advanced HER2-positive breast cancer previously treated with trastuzumab emtansine: a dose-expansion, phase 1 study. Lancet Oncol. 2019;20(6):816-826.

47. Ogitani Y, Hagihara K, Oitate M, Naito H, Agatsuma T. Bystander killing effect of DS-8201a, a novel anti-human epidermal growth factor receptor 2 antibody-drug conjugate, in tumors with human epidermal growth factor receptor 2 heterogeneity. Cancer Sci. 2016;107(7):1039-1046.

48. Ogitani Y, Aida T, Hagihara K, et al. DS-8201a, A novel HER2-targeting ADC with a novel DNA topoisomerase i inhibitor, demonstrates a promising antitumor efficacy with differentiation from T-DM1. Clin Cancer Res. 2016;22(20):5097-5108.

49. Okamoto H, Oitate M, Hagihara K, et al. Pharmacokinetics of trastuzumab deruxtecan (T-DXd), a novel anti-HER2 antibody-drug conjugate, in HER2-positive tumour-bearing mice. Xenobiotica. 2020;50 (10):1242-1250.

50. Nagai Y, Oitate M, Shiozawa H, Ando O. Comprehensive preclinical pharmacokinetic evaluations of trastuzumab deruxtecan (DS-8201a), a HER2-targeting antibody-drug conjugate, in cynomolgus monkeys. Xenobiotica. 2019;49(9):1086-1096. 
51. Daiichi Sankyo Inc. Enhertu (Fam-Trastuzumab Deruxtecan-Nxki): US Prescribing Information; 2019.

52. Takegawa N, Tsurutani J, Kawakami H, et al. [fam-] trastuzumab deruxtecan, antitumor activity is dependent on HER2 expression level rather than on HER2 amplification. Int J Cancer. 2019;145(12):3414-3424.

53. Bartsch R. Trastuzumab-deruxtecan: an investigational agent for the treatment of HER2-positive breast cancer. Expert Opin Investig Drugs. 2020;29(9):901-910.

54. Takegawa N, Nonagase Y, Yonesaka K, et al. DS-8201a, a new HER2-targeting antibody-drug conjugate incorporating a novel DNA topoisomerase I inhibitor, overcomes HER2-positive gastric cancer T-DM1 resistance. Int $J$ Cancer. 2017;141 (8):1682-1689.

55. Tsurutani J, Iwata H, Krop I, et al. Targeting HER2 with trastuzumab deruxtecan: a dose-expansion, Phase I Study in multiple advanced solid tumors. Cancer Discov. 2020;10:5.

56. Smit EF, Nakagawa K, Nagasaka M, et al. Trastuzumab deruxtecan (T-DXd; DS-8201) in patients with HER2-mutated metastatic non-small cell lung cancer (NSCLC): interim results of DESTINY-Lung01. J Clin Oncol. 2020;38(15_suppl):9504.

57. Horvath L, Pircher A. ASCO 2020 non-small lung cancer (NSCLC) personal highlights. Magazine Eur Med Oncol. 2021;14(1):66-69.

58. Nakagawa K, Nagasaka M, Felip E, et al. OA04.05 trastuzumab deruxtecan in HER2-overexpressing metastatic non-small cell lung cancer: interim results of DESTINY-Lung01. J Thor Oncol. 2021;16:3.

59. Modi S, Park H, Murthy RK, et al. Antitumor activity and safety of trastuzumab deruxtecan in patients with HER2-low-expressing advanced breast cancer: results from a Phase IB Study. $J$ Clin Oncol. 2020;38(17):1887-1896.

60. Borghaei H, Besse B, Bardia A, et al. Trastuzumab deruxtecan (T-DXd; DS-8201) in combination with pembrolizumab in patients with advanced/metastatic breast or non-small cell lung cancer (NSCLC): a phase Ib, multicenter, study. J Clin Oncol. 2020;38 (15_suppl):TPS1100-TPS1100.
61. Planchard D, Yang JC-H, Brahmer JR, et al. 185TiP A phase Ib dose-escalation study evaluating trastuzumab deruxtecan (T-DXd) and durvalumab in combination with chemotherapy as first-line treatment in patients with advanced or metastatic nonsquamous non-small cell lung cancer (NSCLC) and HER2 overexpression (DESTINY-Lung03. $J$ Thor Oncol. 2021;16:4.

62. Garon EB, Ciuleanu TE, Arrieta O, et al. Ramucirumab plus docetaxel versus placebo plus docetaxel for second-line treatment of stage IV non-small-cell lung cancer after disease progression on platinum-based therapy (REVEL): a multicentre, double-blind, randomised Phase 3 trial. Lancet. 2014;384(9944):665-673.

63. Yagisawa M, Nakamura Y, Yoshino T, et al. A basket trial of trastuzumab deruxtecan, a HER2-targeted antibody-drug conjugate, for HER2-amplified solid tumors identified by circulating tumor DNA analysis (HERALD trial). $J$ Clin Oncol. 2020;38(15_suppl): TPS3650-TPS3650.

64. Elamin Y, Robichaux J, Lam V, et al. OA 12.01 the preclinical and clinical activity of poziotinib, a potent, selective inhibitor of EGFR Exon 20 mutant NSCLC. J Thor Oncol. 2017;12(11):S1776-undefined.

65. Heymach J, Negrao M, Robichaux J, et al. OA02.06 a phase II trial of poziotinib in EGFR and HER2 exon 20 mutant Non-Small Cell Lung Cancer (NSCLC). J Thor Oncol. 2018;13(10):S323-S324.

66. Le X, Goldman J, Clarke J, et al. Abstract CT081: poziotinib activity and durability of responses in previously treated EGFR exon 20 NSCLC patients - a Phase 2 study. Cancer Res. 2020;80(16 Supplement):CT081 LP-CT081.

67. Robichaux JP, Elamin YY, Vijayan RSK, et al. Pan-cancer landscape and analysis of ERBB2 mutations identifies poziotinib as a clinically active inhibitor and enhancer of T-DM1 activity. Cancer Cell. 2019;36:4.

68. Liu SV, Villaruz LC, Lee VHF, et al. LBA61 First analysis of RAIN701: study of tarloxotinib in patients with non-small cell lung cancer (NSCLC) EGFR Exon 20 insertion, HER2-activating mutations \& other solid tumours with NRG1/ERBB gene fusions. Ann Oncol. 2020;31:S1189-undefined.
Lung Cancer: Targets and Therapy

\section{Publish your work in this journal}

Lung Cancer: Targets and Therapy is an international, peerreviewed, open access journal focusing on lung cancer research, identification of therapeutic targets and the optimal use of preventative and integrated treatment interventions to achieve improved outcomes, enhanced survival and quality of life for the cancer patient. Specific topics covered in the journal include: Epidemiology,

\section{Dovepress}

detection and screening; Cellular research and biomarkers; Identification of biotargets and agents with novel mechanisms of action; Optimal clinical use of existing anticancer agents, including combination therapies; Radiation and surgery; Palliative care; Patient adherence, quality of life, satisfaction; Health economic evaluations. 